- the struggle for full and equal life for people with rheumatic and musculoskeletal diseases

- the establishment of regional clubs and specific patient groups of Czech League Against Rheumatism

Objectives: Creating a campaign Rheumatism Challenge 2018 - "Rheumatism in motion" We are going in that together! The aim of this campaign is improving the health condition of people with RMDs. To motivate patients to move regularly and raising patient awareness about the importance of physical activity.

Methods: Walking as a means to reach a goal is the main content of this campaign. The aim of each participant in this campaign is to reach as much as possible steps and kilometers every months. Exiting challenges prompts patients to higher goals and giving them courage. Through walking Czech League Against Rheumatism wants to improve physical and mental health of people with RMDs. Wants to maintain a joint mobility and reduce a pain. Know and find the right kind of movement for each patient. Information about this campaign is published on the webpage, Facebook and Instagram of Czech League Against Rheumatism.

Results: This campaign managed to raise awareness about the importance of physical activity in patients with rheumatic and musculoskeletal diseases among the general public. Information about the campaign was in press and television. The campaign continues in 2019.

In response to the campaign has increased the number of members in Czech League Against Rheumatism, an increased number of visitors and fans of the Facebook page Czech League Against Rheumatism.

Conclusion: This campaign seems to be very useful in promoting how important physical activity is for patients with RMDs. This campaign can support patients be more healthy and physically active. Active approach to one another life can inspire other patients and their families and friends. Very important is also the support of this campaign from the professional community, medical doctors and health professionals.

Disclosure of Interests: None declared

DOI: 10.1136/annrheumdis-2019-eular.3819

\section{PARE0008 UNDERSTANDING THE IMPACT OF ANKYLOSING SPONDYLITIS IN DAILY LIFE OF THE PORTUGUESE PATIENTS: RESULTS FROM THE ASSESSMENT OF RESULTS IN ANKYLOSING SPONDYLITIS (AREA) STUDY}

Filipe Araújo ${ }^{1}$, Claudia Duarte ${ }^{2}$, Jorge Brandão ${ }^{3}$, Rui Cernadas ${ }^{4}$, Elsa Mateus ${ }^{5}$, Luís Cunha Miranda ${ }^{6}$, José Canas Da Silva ${ }^{7}$, José Gomes Da Silva ${ }^{8}$, Pedro Simões Coelho ${ }^{2}$, Catarina Marques ${ }^{9} .{ }^{1}$ Hospital Sant'Ana, Rheumatology and Osteoporosis Unit, Lisbon, Portugal; ${ }^{2}$ NOVA IMS Information Management School, Lisbon, Portugal; ${ }^{3}$ APMGF - Associação Portuguesa de Medicina Geral e Familiar, Delegação Distrital de Coimbra, Coimbra, Portugal; ${ }^{4}$ Serviços Médicos Continental, Lisbon, Portugal; ${ }^{5}$ Liga Portuguesa Contra as Doenças Reumáticas, Lisboa, Portugal; ${ }^{6} I P R$ - Portuguese Institute of Rheumatology, Lisbon, Portugal; ${ }^{7}$ Hospital Garcia de Horta, Lisboa, Portugal; ${ }^{8}$ National Association of Ankylosing Spondylitis, Oeiras, Portugal; ${ }^{9}$ Portuguese League Against Rheumatic Diseases, Lisbon, Portugal

Background: AS is known to have a significant burden upon the individual, family and society. It is essential to assess the impact of AS in patients' lives, to raise awareness and improve early referral, diagnosis and treatment, for a better quality of life.

Objectives: To assess the impact of AS in the life of Portuguese patients (work, daily activities, social life).

Methods: The Assessment of REsults in Ankylosing spondylitis (arEA) study was developed by the NOVA-IMS in cooperation with: Portuguese Society of Rheumatology, Portuguese Association of Family Physicians, National Association of Primary Care Units, National Association of AS Patients and the Portuguese League Against Rheumatic Diseases. The arEA aimed at assessing reasons for delayed referral of suspicious cases of AS to the rheumatologist, as well as disease impact in patients' lives, global health and work. A comprehensive online survey was developed with the collaboration of LPCDR and sent to AS patients. Data on demographics, lifestyle habits, daily life activities, working habits, disease indexes and healthcare utilization, health status/quality of life indicators, EQ-5D and BASDAI scales. A generalized linear model was adjusted in order to identify the factors impacting on quality of life of patients.

Results: 354 patients responded the survey, $42.1 \%$ female, most frequently from the 35-44 year-old age group. Mean age at disease onset was 27 years old, while the diagnosis was confirmed 7 years later. In the previous 12 months, the average of working days lost due to AS issues was 37 (including sick leaves). During that period, AS has affected work productivity in average around 73 days. Cumulatively, patients' relatives or friends had also lost about 13 days of work, to provide them assistance. Regarding limitations in daily life activities, house cleaning $(55.5 \%)$, physical exercise $(46.5 \%)$, getting in/out of bed $(45.6 \%)$, using stairs $(37.9 \%)$, shopping $(37.4 \%)$ and tying shoes $(35.2 \%)$ were reported as being highly limited by AS. As for the social life, family and friends' relationships were reported as not being affected by over $60 \%$ of AS patients. However, $19.8 \%$ of the respondents have reported a worse relationship with their partners after diagnosis, and $29.7 \%$ reported having decreased the frequency of sexual intercourse. Leisure activities, were only reported as not being affected by $34.6 \%$ and $40.5 \%$ of patients, while the practice of sports has much decreased in $40.7 \%$ of the respondents. Almost $80 \%$ had mobility issues during the last year, and over $65 \%$ had their usual activities/routines affected by AS. The average BASDAI score is 5.5 and the EQ visual analogue scale 55. Analyzing the impact of treatment, biologics and biosimilars were reported as having significantly improved patients' quality of life, except for physical or sexual activity.

Conclusion: AS has a major impact in the daily life of patients, work productivity, social relationships and leisure activities. Early diagnosis and treatment, along with physical rehabilitation/exercise plans, can reduce this impact and improve their quality of life.

Disclosure of Interests: None declared

DOI: 10.1136/annrheumdis-2019-eular.6277

\section{Best practice campaigning}

\section{PARE0009 THE PHYSICAL AND EMOTIONAL BURDEN OF RHEUMATOID ARTHRITIS: DATA FROM RA MATTERS, A WEB-BASED SURVEY OF PATIENTS AND PHYSICIANS IN EUROPE AND CANADA}

Rieke Alten ${ }^{1}$, Neil Betteridge ${ }^{2}$, Francesco de Leonardis ${ }^{3}$, Nicole Tietz ${ }^{3}$, Mariana Guerreiro ${ }^{3}$, Mart van de Laar ${ }^{4} .{ }^{1}$ Schlosspark Klinik University Médicine, Berlin, Germany, ${ }^{2}$ Neil Betteridge Associates, London, United Kingdom; ${ }^{3}$ Eli Lilly and Company, Indianapolis, United States of America; ${ }^{4}$ Arthritis Centre Twente, Medisch Spectrum Twente, Enschede, Netherlands

Background: Rheumatoid Arthritis (RA) is a chronic inflammatory disorder that negatively affects the patients' health-related quality of life (QOL). Despite much progress in addressing the burden of RA, a gap exists in terms of understanding 'what really matters' to patients with RA, and balancing patients' QOL aspirations with clinical targets in key treatment decisions.

Objectives: This international survey investigated how RA affects the lives of patients according to the perceptions of both patients and healthcare providers (HCPs), in order to help health professionals attune their care on the domains that really matter to patients.

Methods: Data were collected from patients with RA, and rheumatologists or HCPs who treat RA in Canada, France, Germany, Italy, Netherlands, Spain, Sweden, and UK using a structured, closed-ended questionnaire in their local language. Respondents for the survey were recruited from survey panels of verified unique responses, as well as through social media. Data were summarized in terms of both frequency and percentages of patients to understand the experience of patients living with RA on daily activities, relationships, work, and aspirations.

Results: Overall, 5400 adult patients $(81 \%$ female; mean age 52 years), and 808 rheumatologists or HCPs participated in the survey between November 2016 and February 2017. Of these, the highes proportion of patient and HCP respondents were from the UK $(n=1250)$ and France $(n=230)$, respectively. Overall, $52 \%$ of patients and $62 \%$ of HCPs believed that the emotional impact of RA is not well understood by people without the disease; whereas $46 \%$ of patients and $52 \%$ of HCPs had a similar belief with regards to the physical impact of the disease. Patients who felt that the impact of RA is less well understood by others expressed more negative feelings about every aspect of their life with RA. Important relationships with spouse or partner, children, family, friends, and colleagues were generally affected negatively. Almost half of the patients $(48 \%)$ who participated in the survey reported that they were forced to take long-term leave/retirement or experienced slow career progress since being diagnosed with $R A$; more than 3 in 5 patients found exercising to be difficult; and almost 1 in 4 patients found difficulties in taking care of personal grooming. Aching/stiff joints, pain, and fatigue were the key physical barriers to activities for patients, and $65 \%$ of patients felt frustrated when they were unable to undertake or complete daily activities due to their disease. Two-thirds of patients wished to be able to accept their life with RA and do what they can to cope with it in the future. Over half of 
the patients $(52 \%)$ had a hope that the physical impact of RA will be better understood in the future.

Conclusion: Despite major advancements in the treatment of RA, the chronic disease continues to significantly affect many aspects of patients' lives, including relationships, career progression, daily activities, and ability to work. Both patients and HCPs felt that the physical and emotional impact of RA is not well understood by people without the disease. In line with a recently published study, pain, fatigue, and physical function remain primary barriers for patients to live a normal life and to participate fully. $^{2}$ In RA treatment decisions, patients' personal goals and patientreported outcomes should be given greater consideration along with clinical targets.

\section{REFERENCES}

[1] Wysocka-Skurska I, et al. Clin Interv Aging. 2016;11:1741-50.

[2] Oude Voshaar MAH, et al. Arthritis Care Res (Hoboken). 2018 Oct 24. doi: 10.1002/acr.23799. [Epub ahead of print].

Disclosure of Interests: Rieke Alten Grant/research support from: BristolMyers Squibb, Speakers bureau: Bristol-Myers Squibb, Neil Betteridge Consultant for: Amgen, Eli Lilly, Grunenthal, GSK, Heart Valve Voice, Janssen, Roche, Sanofi Genzyme and Sanofi Regeneron, Speakers bureau: Amgen, Eli Lilly, Grunenthal, GSK, Heart Valve Voice, Janssen, Roche, Sanofi Genzyme and Sanofi Regeneron, Francesco de Leonardis Shareholder of: Eli Lilly and Company, Employee of: Eli Lilly and Company, Nicole Tietz Shareholder of: Eli Lilly and Company, Employee of: Eli Lilly and Company, Mariana Guerreiro Shareholder of: Eli Lilly and Company, Employee of: Eli Lilly and Company, Mart van de Laar Grant/research support from: Eli Lilly, Pfizer, Merck, AbbVie and Janssen Cilag, Consultant for: Sanofi Genzym, Eli Lilly, Pfizer, Merck, Abbvie and Janssen Cilag, Speakers bureau: Eli Lilly, Pfizer and Janssen Cilag

DOI: 10.1136/annrheumdis-2019-eular.907

\section{PARE0010 INSIDE OUT - A PHOTO CAMPAIGN TO CREATE AWARENESS FOR YOUNG PEOPLE WITH RHEUMATIC AND MUSCULOSKELETAL DISEASES}

Meike Voeten ${ }^{1}$, Elke van Delft ${ }^{1,2}$, Aimee Haenen ${ }^{1}$, Wendy Olsder ${ }^{1} .{ }^{1}$ Youth- $R$-Well. com, Eindhoven, Netherlands; ${ }^{2}$ Maasstad hospital, Department of rheumatology, Rotterdam, Netherlands

Background: Inside Out is a photo campaign of Youth-R-Well.com, the organisation for young people (16-30 years) with rheumatic and musculoskeletal diseases (RMDs) in the Netherlands. The project was inspired by Unga Reumatiker, the youth organisation in Sweden and their campaign \#doesntshowdoesntexist. The campaign of Unga Reumatiker showed that young people can suffer from rheumatic diseases, even though these diseases are invisible. Youth-R-Well.com recognized that the invisibility of an RMD can have a major impact on the lives of young people and wanted to create a similar photo campaign to show the invisible side of RMDs.

Objectives: The main objective of this project was to create more awareness for rheumatic and musculoskeletal diseases affecting young people. One of the hardest parts of living with an RMD is the invisibility of the disease in daily life. We wanted to show that even though you cannot see it, many young people have to deal with the consequences of having an RMD on a daily basis. We also wanted to show other young people who are in a similar situation, that they are not the only one. Methods: To make the invisible side of an RMD visible for others, Youth-R-Well.com created Inside Out, an eight part photo campaign. Eight young people with different types of RMDs, took part in this project. From each individual, two photos were taken: one photo in daylight and one photo in the dark with their rheumatic-spots highlighted by blacklight paint. Next to the photos, the participants introduced themselves in a few sentences and ended with a life quote with a focus on the positive side of life. They were all telling very different and very personal stories. The general quote of the photo campaign was "Although you cannot see it from the outside, it is still there". The photo campaign was posted on Facebook and Instagram. The photo campaign was released the week before World Arthritis Day, in which the photos of one person were shared on each day. The final photo, a group photo with all the young people and their black light photos, was shared on World Arthritis Day 12 October 2018.

Results: It was a successful campaign, in which we reached almost 30.000 people on Facebook and 5.000 people on Instagram. The success of the project and all the shares and great comments it received were overwhelming. The power of two photos next to each other had a large impact on people who were not familiar with the fact that RMDs do exist among young people. As a result of the great comments, this photo campaign is going to be exhibited in Reade, the center for Rehabilitation and Rheumatology in Amsterdam, to reach more people and make them more aware of the invisibility of RMDs.

Conclusion: Based on inspiration from a youth organisation in Sweden Youth-R-Well.com created the successful campaign Inside Out. We managed to create more awareness for young people with RMDs and the invisible side of having an RMD. We will continue to spread this campaign and show that young people with RMDs are not alone.

Acknowledgement: First of all, we want to thank all our brave participants who took part in this campaign. Secondly, we want to thank Unga Reumatiker, who inspired Youth-R-Well.com to launch this amazing photo campaign.

Disclosure of Interests: None declared

DOI: 10.1136/annrheumdis-2019-eular.5439

\section{Innovations in arthritis health care}

\section{PARE0011 PILOTING THE FIRST PATIENT PARTNERS PROGRAM FOR AXIAL SPONDYLOARTHRITIS: THE JOURNEY OF BELGIAN PATIENTSTO BECOME EXPERT TEACHERS IN RHEUMATOLOGY}

Jahel Roels ${ }^{1}$, Philip Gadeyne ${ }^{1}$, Luc Ghekiere ${ }^{1}$, Liliane Ovaere ${ }^{1}$, Thijs Swinnen ${ }^{2}$, Philippe Carron ${ }^{3}{ }^{1}$ Patient Partners Program Belgium vzw asbl, PPP-SPA, SintJoris-Weert, Belgium; ${ }^{2}$ University Hospitals Leuven, Division of Rheumatology, Leuven, Belgium; ${ }^{3}$ University Hospital Ghent, Rheumatology, Gent, Belgium

Background: Patients with axial spondyloarthritis $(\operatorname{axSpA})$ were trained to deliver experience-based workshops to medical students, general practitioners, physiotherapists and other healthcare providers concerning axSpA The most important aims of these workshops are to improve the early diagnosis and to increase awareness of axSpA in the medical sector. Taking an expert role as a patient however requires an extensive training.

Objectives: To describe the training program in detail and to illustrate both the less successful and the optimal implementation steps taken thus far.

Methods: In its first iteration, the training program was developed as a resource book including a curriculum, significant amounts of homework, without enough time for practical exercises. This resulted in a drop-out of a large portion of trainees. Thereafter, the training method has been adapted by copying successful parts of the approach of The Patient Partner ${ }^{\circledR}$ Program for Rheumatoid Arthritis (launched in 1999). Also, more concrete training opportunities via a DVD demonstrating the complete course and training materials, as well as offering hands-on trainings during a three day overnight stay were added. Besides creating opportunities for intensive practicing with each other, there was also more time for informal contacts and it allowed for proper group dynamics. So there is a lot of time for sharing among patients with a similar health background. A comfortable environment was created for the candidates, enabling them to go through a complete process and giving them sufficient time to train all the components of the program.

Results: On February 22nd 2018, five fresh SpA-patients started the training, all five of them successfully completed the training on June 2nd 2018. On a first evening of familiarization, trainers tried to know more about the motivation of the trainees, while in an individual interview a rheumatologist reassured that the candidates have the correct diagnosis, as their story should be consistent. The selected candidates received part 1 of the training manual by mail and they were invited to profoundly study the terminology and the anatomy, related to their disease.

During the first full day of the training, the trainers took time to explain this terminology and anatomy. Later on this constituted the building blocks of the course. The trainers closed this first day demonstrating the history taking and they asked the trainees to study at home their personal medical file.

On day 2, the homework of the previous day was first discussed, followed by related exercises. Day 2 finished with the demonstration of the clinical examination.

The training program was completed with three days with overnight stay. During these days a lot of practical exercises of the clinical examination were organized, followed by a discussion of the impact of the disease and the importance of active participation. The program ended with a general rehearsal and a written as well as an oral test to evaluate how each trainee had assimilated all this. 